\title{
Large Volume Limit of the Distribution of Characteristic Exponents in Turbulence
}

David Ruelle

I.H.E.S., F-91440 Bures-sur-Yvette, France

\begin{abstract}
For spatially extended conservative or dissipative physical systems, it appears natural that a density of characteristic exponents per unit volume should exist when the volume tends to infinity. In the case of a turbulent viscous fluid, however, this simple idea is complicated by the phenomenon of intermittency. In the present paper we obtain rigorous upper bounds on the distribution of characteristic exponents in terms of dissipation. These bounds have a reasonable large volume behavior. For two-dimensional fluids a particularly striking result is obtained: the total information creation is bounded above by a fixed multiple of the total energy dissipation (at fixed viscosity). The distribution of characteristic exponents is estimated in an intermittent model of turbulence (see [7]), and it is found that a change of behavior occurs at the value $D=2.6$ of the self-similarity dimension.
\end{abstract}

\section{To Freeman J. Dyson, Res Jost, and Arthur S. Wightman}

The relation between physics - real physics - and mathematics - real mathematics has not been an easy one in the last thirty years. It took vision to see that this relation is possible and fruitful now as it was in the times of Archimedes, Newton, and Einstein. Res Jost in Zürich, Freeman Dyson and Arthur Wightman in Princeton had that vision, and made many others share it.

\section{Introduction}

The time evolutions of relevance to physics often define nonlinear differentiable dynamical systems of the form

$$
\frac{d x}{d t}=F(x),
$$

where $x$ varies in a vector space or manifold which may have infinite dimension. Let the initial condition $x_{0}$ for $(0.1)$ be replaced by $x_{0}+\delta x_{0}$; if the following limit exists

$$
\mu\left(x_{0}, \delta x_{0}\right)=\lim _{t \rightarrow \infty} \frac{1}{t} \log \left\|\delta x_{t}\right\|,
$$


it is called a characteristic exponent ${ }^{1}$. The multiplicative ergodic theorem of Oseledec $^{2}$ asserts that $\mu\left(x_{0}, \delta x_{0}\right)$ is well defined for almost every $x_{0}$ (and all $\delta x_{0}$ ) with respect to any invariant probability measure $\varrho$ under time evolution. If $\varrho$ is ergodic, the characteristic exponents are independent of $x_{0} \varrho$-almost everywhere. The presence of a characteristic exponent $\chi>0$ indicates sensitive dependence on initial condition.

We may call spectrum the set of characteristic exponents associated with an ergodic measure $\varrho$ (with multiplicities to be defined later). In the case of a Hamiltonian system, and taking for $\varrho$ an ergodic component of the Liouville measure, one finds that the spectrum is invariant under change of sign. For a dissipative system, the choice of invariant measure is not obvious, and there is no invariance of the spectrum under sign change.

For certain classes of physical systems a large volume limit exists (for equilibrium statistical mechanics this is the thermodynamic limit). We want to investigate the possibility of defining a large volume limit for certain dynamical systems. The idea is that, if several systems with independent dynamics occupy disjoint regions $\Lambda_{1}, \ldots, \Lambda_{N}$, the spectrum of the joint system is simply the union of the spectra of the subsystems (repeated characteristic exponents appear with added multiplicity). If an interaction between the subsystems is introduced, one may hope that this does not alter the spectrum much and that, in the large volume limit, a number of characteristic exponents per unit volume may be defined.

In our present state of knowledge, it seems difficult to prove the existence of a large volume limit for the spectrum of nontrivial dynamical systems. If that could be done, one would like to know, for instance, if the density of the spectrum is finite or infinite at 0 (i.e. for infinite relaxation times). In the case of a hamiltonian system of hard spheres, Sinai [32] has obtained a lower bound to the sum of the positive characteristic exponents divided by the volume. In this paper we shall be concerned with viscous fluids. We shall on one hand give rigorous upper bounds on the spectrum, and on the other hand estimate the distribution of characteristic exponents in an intermittent model of turbulence. Because of intermittence, taking the large volume limit is more delicate than one might imagine, but a simple answer is nevertheless obtained.

\section{Characteristic Exponents for the Navier-Stokes Equation}

Let $\Omega$ be a bounded domain in $\mathbb{R}^{d}(d=2$ or 3$)$. We assume that the time evolution of the velocity field $\mathbf{v}=\left(v_{1}, \ldots, v_{d}\right)$ of a viscous fluid contained in $\Omega$ is given by the Navier-Stokes equation

$$
\frac{\partial \mathbf{v}}{\partial t}+\sum_{i} v_{i} \frac{\partial \mathbf{v}}{\partial x_{i}}=v \Delta \mathbf{v}-\nabla p+\mathbf{g},
$$

1 We have written $\delta x$ as an infinitesimal increment of $x$; more properly $\delta x$ should be considered as a "tangent vector," so that $\delta x_{t}=\frac{\partial x_{t}}{\partial x_{0}} \delta x_{0}$

2 See Oseledec [22], Raghunathan [23], Ruelle [29], and Mañé [19] 
supplemented by the incompressibility condition

$$
\nabla \cdot \mathbf{v}=0
$$

In (1.1), $v$ is the kinematic viscosity, $p$ the pressure and $\mathbf{g}$ an external force. One takes (1.2) into account by projecting (1.1) on a space of divergence free vector fields; this has in particular the effect of eliminating the pressure term $-\nabla p$. External action on the fluid is obtained either via $\mathbf{g}$, or by imposing a nonzero velocity at the boundary of $\Omega$. We follow Foias and Temam [6] in assuming that $\partial \Omega$ is of class $C^{2}$ consisting of a finite number of connected components, and that $\Omega$ is locally on one side of $\partial \Omega$. The external force $\mathbf{g}$ is assumed to be square integrable in $\Omega$, and time independent. The velocity $\phi$ imposed on the boundary is tangent to $\partial \Omega$, independent of $t$, and is assumed to extend to a divergence free vector field with square integrable second derivatives in $\Omega$. We shall assume that a probability measure $\varrho$ with compact support in a suitable space of divergence free vector fields is given so that it is invariant under the time evolution given by (1.1) and (1.2).

If $d=2$, there are good existence and uniqueness theorems for solutions of the Navier-Stokes equation, but if $d=3$, we have to assume that time evolution is well defined in a neighborhood of the support of $Q^{3}$. One can then write a linear "variational equation" of the form

$$
\frac{d \varphi}{d t}=\mathscr{K}(\mathbf{v}(t)) \varphi
$$

for the time evolution of an infinitesimal increment $\boldsymbol{\varphi}=\delta \mathbf{v}$ of $\mathbf{v}$ (more appropriately, $\varphi$ is considered as a tangent vector).

Even though we won't go into the details of Navier-Stokes theory, we want to describe more precisely the Hermitian part $\mathscr{H}(\mathbf{v}(t))$ of the operator $\mathscr{K}(\mathbf{v}(t))$ appearing in (1.3). Let $D$ be the closure of the space of real $C^{\infty}$ functions with compact support in $\Omega$ with respect to the Dirichlet norm

$$
\|\varphi\|_{D}=\left[\int \sum_{i}\left(\partial_{i} \varphi(x)\right)^{2} d x\right]^{1 / 2}
$$

Then $D$ is a Hilbert space and $D C L^{2}=L^{2}(\Omega, d x)$. We denote by $D^{d} C L^{2} \otimes \mathbb{R}^{d}$ the corresponding space of vector fields, with norm $\|\boldsymbol{\varphi}\|=\left(\sum_{j=1}^{d}\left\|\varphi_{j}\right\|^{2}\right)^{1 / 2}$, and by $\mathscr{D}$ (respectively $\mathscr{L}$ ) the subspace of $D^{d}$ (respectively $L^{2} \otimes \mathbb{R}^{d}$ ) consisting of divergencefree vector fields. We denote by $P$ the orthogonal projection $L^{2} \otimes \mathbb{R}^{d} \mapsto \mathscr{L}$. The unbounded self-adjoint operator $\mathscr{H}(\mathbf{v})$ will be the restriction to $\mathscr{L}$ of $P H(\mathbf{v})$ where the operator $H(\mathbf{v})$ on $L^{2} \otimes \mathbb{R}^{d}$ is defined at first formally by

$$
(H(\mathbf{v}) \boldsymbol{\varphi})_{j}=v \Delta \varphi_{j}-\frac{1}{2} \sum_{i}\left(\frac{\partial v_{i}}{\partial x_{j}}+\frac{\partial v_{j}}{\partial x_{i}}\right) \varphi_{i} .
$$

3 For a discussion of the Navier-Stokes equation, see the monographs of Ladyzhenskaya [11], Lions [15], Temam [33], and Girault and Raviart [9]. A review is given in Foias and Temam [6], and a discussion from the point of view relevant here in Ruelle [28] 
The $\mathbf{v}$ which will occur belong to the support of $\varrho$ and may be assumed to have square integrable derivatives (up to second order) in $\Omega ; v(t)$ depends continuously on $t$ (in fact real analytically) with respect to the $L^{2}$ topology of the derivatives, and supp $\varrho$ is compact for this topology ${ }^{4}$.

The symmetric quadratic form

$$
(\varphi, \psi) \mapsto \beta(\varphi, \psi)=\int_{\Omega} d x \varphi(x) \frac{\partial v_{i}}{\partial x_{j}} \psi(x)
$$

on $D \times D$ satisfies

$$
|\beta(\varphi, \varphi)| \leqq\left\|\frac{\partial v_{i}}{\partial x_{j}}\right\|_{L^{2}}\left(\|\varphi\|_{L^{4}}\right)^{2}
$$

where

$$
\begin{aligned}
\|\varphi\|_{L^{4}}^{2} & \leqq\|\varphi\|_{L^{2}}^{1 / 2}\|\varphi\|_{L^{6}}^{3 / 2} \leqq \frac{1}{2}\left(\alpha^{2}\|\varphi\|_{L^{6}}^{2}+\frac{1}{\alpha^{2}}\|\varphi\|_{L^{6}}\|\varphi\|_{L^{2}}\right) \\
& \leqq \frac{3}{4} \alpha^{2}\|\varphi\|_{L^{6}}^{2}+\frac{1}{4 \alpha^{6}}\|\varphi\|_{L^{6}}^{2} .
\end{aligned}
$$

On the other hand, by Sobolev type properties ${ }^{5}$ there are continuous embeddings $D \subset L^{r}(\Omega, d x)$ for each $r<\infty$ if $d=2$, and $D \subset L^{6}(\Omega, d x)$ if $d=3$. Thus, given $a>0$, there is $b>0$ such that

$$
|\beta(\varphi, \varphi)| \leqq a\|\varphi\|_{D}^{2}+b\|\varphi\|_{L^{2}}^{2} .
$$

If $\Delta$ denotes the Dirichlet Laplacian, (1.5) expresses that the operator of multiplication by $\frac{\partial v_{i}}{\partial x_{j}}$ is form bounded with respect to $\Delta$. Therefore $H(\mathbf{v})$ and $\mathscr{H}(\mathbf{v})$ have natural definitions as self-adjoint operators corresponding to quadratic forms on $D^{3}$ and $\mathscr{D}$ respectively ${ }^{6}$.

The Cwikel-Lieb-Rosenbljum theorem (see below) will imply that the spectrum of $\mathscr{H}(\mathbf{v})$ is discrete. We denote by $a^{(1)}(\mathbf{v}) \geqq a^{(2)}(\mathbf{v}) \geqq \ldots$ the eigenvalues of $\mathscr{H}(\mathbf{v})$ repeated according to multiplicity. Since

$$
a^{(1)}(\mathbf{v})=\{\max (\boldsymbol{\varphi}, \mathscr{H}(\mathbf{v}) \boldsymbol{\varphi})=\boldsymbol{\varphi} \in \mathscr{D} \quad \text { and } \quad(\boldsymbol{\varphi}, \boldsymbol{\varphi})=1\},
$$

the above estimates imply that $a^{(1)}(\mathbf{v})$ depends continuously on $\mathbf{v}$ with respect to the $L^{2}$ norm of $\frac{\partial v_{i}}{\partial x_{j}}$. Replacing $\mathscr{H}$ by $\mathscr{H}^{\wedge n}$ acting on the exterior power $\mathscr{L}^{\wedge n}$ (see below), one sees that $\sum_{1}^{n} a^{(k)}(\mathbf{v})$ also depends continuously on $\mathbf{v}$. Therefore the eigenvalues of $\mathscr{H}(\mathbf{v}(t))$ depend continuously on $t$ when $v(0)$ belongs to the support of $\varrho$.

4 See Foias and Temam [6] and Ruelle [28]

5 See for instance Reed and Simon [24, II, pp. 112 and 113]

6 This is a consequence of what Reed and Simon call the KLMN theorem (see Theorem X.17 in $[24, \mathrm{II}])$ 
Let $\mu^{(1)}\left(\mathbf{v}_{0}\right) \geqq \mu^{(2)}\left(\mathbf{v}_{0}\right) \geqq \ldots$ be the characteristic exponents, repeated according to multiplicity, and corresponding to the initial condition $\mathbf{v}_{0}$ (where defined). We also write

$$
\mu^{(k)}(\varrho)=\int \varrho\left(d \mathbf{v}_{0}\right) \mu^{(k)}\left(\mathbf{v}_{0}\right)
$$

(remember that if the invariant probability measure $\varrho$ is ergodic, $\mu^{(k)}$ is $\varrho$-almost everywhere constant). The sum of the largest $n$ characteristic exponents $\mu^{(k)}\left(\mathbf{v}_{0}\right)$ can be estimated from the rate of growth of an element $\varphi^{(1)}(t) \wedge \ldots \wedge \varphi^{(n)}(t)$ of the (Hilbert space) exterior power $\mathscr{L}^{\wedge n}$ (see Ruelle [29] or Mañé [19]). We have

$$
\sum_{1}^{n} \mu^{(k)}\left(\mathbf{v}_{0}\right) \geqq \lim _{t \rightarrow \infty} \frac{1}{t} \log \left\|\boldsymbol{\varphi}^{(1)}(t) \wedge \ldots \wedge \boldsymbol{\varphi}^{(n)}(t)\right\|
$$

with equality for suitable $\varphi^{(1)}(0), \ldots, \varphi^{(n)}(0)$ (in fact most choices of initial vectors). Notice that

$$
\begin{aligned}
\frac{d}{d t} & \log \|\left(\boldsymbol{\varphi}^{(1)}(t) \wedge \ldots \wedge \varphi^{(n)}(t) \|\right. \\
& =\frac{1}{2} \frac{d}{d t} \log \left(\boldsymbol{\varphi}^{(1)}(t) \wedge \ldots \wedge \boldsymbol{\varphi}^{(n)}(t), \boldsymbol{\varphi}^{(1)}(t) \wedge \ldots \wedge \boldsymbol{\varphi}^{(n)}(t)\right) \\
& =\sum_{k=1}^{n} \frac{\left(\boldsymbol{\varphi}^{(1)}(t) \wedge \ldots \wedge \boldsymbol{\varphi}^{(n)}(t), \boldsymbol{\varphi}^{(1)}(t) \wedge \ldots \wedge \frac{d}{d t} \boldsymbol{\varphi}^{(k)}(t) \wedge \ldots \wedge \boldsymbol{\varphi}^{(n)}(t)\right)}{\left\|\boldsymbol{\varphi}^{(1)}(t) \wedge \ldots \wedge \boldsymbol{\varphi}^{(n)}(t)\right\|^{2}} \\
& =\frac{\left(\boldsymbol{\varphi}^{(1)}(t) \wedge \ldots \wedge \boldsymbol{\varphi}^{(n)}(t), \mathscr{H}(\mathbf{v}(t))^{\wedge n} \boldsymbol{\varphi}^{(1)}(t) \wedge \ldots \wedge \boldsymbol{\varphi}^{(n)}(t)\right)}{\left\|\boldsymbol{\varphi}^{(1)}(t) \wedge \ldots \wedge \boldsymbol{\varphi}^{(n)}(t)\right\|^{2}},
\end{aligned}
$$

where $\mathscr{H}^{\wedge n} \boldsymbol{\varphi}^{(1)} \wedge \ldots \wedge \varphi^{(n)}=\sum_{k} \boldsymbol{\varphi}^{(1)} \ldots \mathscr{H} \varphi^{(k)} \wedge \ldots \wedge \varphi^{(n)}$. Therefore

$$
\frac{d}{d t} \log \left\|\varphi^{(1)}(t) \wedge \ldots \wedge \varphi^{(n)}(t)\right\| \leqq \sum_{1}^{n} a^{(k)}(\mathbf{v}(t)) .
$$

Choosing $\varphi^{(1)}(0), \ldots, \varphi^{(n)}(0)$ so that equality holds in (1.6), we find

$$
\sum_{1}^{n} \mu^{(k)}\left(\mathbf{v}_{0}\right) \leqq \lim _{T \rightarrow \infty} \frac{1}{T} \int_{0}^{T} d t \sum_{1}^{n} a^{(k)}(\mathbf{v}(t)) .
$$

The ergodic theorem therefore yields

$$
\sum_{1}^{n} \mu^{(k)}(\varrho)=\int \varrho\left(d \mathbf{v}_{0}\right) \sum_{1}^{n} \mu^{(k)}\left(\mathbf{v}_{0}\right) \leqq \sum_{1}^{n} \int \varrho\left(d \mathbf{v}_{0}\right) a^{(k)}\left(\mathbf{v}_{0}\right) .
$$

\section{Rigorous Estimates}

An upper bound to the $a^{(k)}(\mathbf{v})$ is obtained if $\mathscr{H}(\mathbf{v})$ is replaced by $H(\mathbf{v})$ (abandoning the restriction to divergence free vector fields), and if the $d \times d$ matrix $\left(W_{i j}(x)\right)$ with

$$
W_{i j}=-\frac{1}{2}\left(\frac{\partial v_{i}}{\partial x_{j}}+\frac{\partial v_{j}}{\partial x_{i}}\right)
$$


is replaced in (1.4) by the diagonal matrix $\left(w \delta_{i j}\right)$, where $w$ is the largest eigenvalue of $\left(W_{i j}\right)$. The operator obtained is of the form

$$
(v \Delta+w) \otimes \mathbb{1}
$$

acting on $L \otimes \mathbb{R}^{d}$; its eigenvalues are the eigenvalues $b^{(l)}$ of $v \Delta+w(\operatorname{acting}$ on $L$ ) repeated $d$ times. In view of results of Lieb, Thirring, Cwikel, and Rosenbljum we have, for $\lambda \geqq 0$,

$$
\sum_{l: b^{(l)}+\lambda \geqq 0}\left(b^{(l)}+\lambda\right)^{\gamma} \leqq L_{\gamma d} \int_{\Omega} d x \frac{(w+\lambda)^{\gamma+d / 2}}{v^{d / 2}},
$$

where the universal constants $L_{\gamma d}$ are finite for $\gamma+d / 2>1$ (see Reed and Simon [24, IV] for a discussion of the Cwikel-Lieb-Rosenbljum theorem and Lieb and Thirring [14] for a conjecture of the best value of the $L_{\gamma d}$ ). In particular

$$
\sum_{k: a^{(k)} \geqq 0}\left(a^{(k)}\right)^{\gamma} \leqq 3 L_{\gamma d} \int_{\Omega} d x \frac{w^{\gamma+d / 2}}{v^{d / 2}} .
$$

We also have

$$
\begin{aligned}
\sum_{l: b(l) \geqq-\lambda} 1 & \geqq\left(\text { number of eigenvalues of }-\Delta \leqq \frac{\lambda}{v}\right) \\
& \approx C_{d}\left(\frac{\lambda}{v}\right)^{d / 2}|\Omega|
\end{aligned}
$$

by the Weyl estimate, where $C_{2}=\frac{1}{4 \pi}, C_{3}=\frac{1}{6 \pi^{2}}$ (we have denoted by $|\Omega|$ the volume of $\Omega$ ). The Weyl estimate assumes $\lambda \rightarrow \infty$ and $\Omega$ fixed (see Reed and Simon [24, IV, Theorem XIII. 78]) but we shall apply it to the equivalent situation where $\lambda$ is fixed and $\Omega \rightarrow \infty$. (The sense in which one has to let $\Omega \rightarrow \infty$ can be read from the proof of Theorem XIII.78 in [24, IV]. For instance, dilations of a fixed shape are allowed.) We have thus

$$
\sum_{l: b^{(l)}+\lambda \geqq 0} b^{(l)} \lesssim L_{1 d} \int_{\Omega} d x \frac{(w+\lambda)^{1+d / 2}}{v^{d / 2}}-C_{d}|\Omega| \frac{\lambda^{1+d / 2}}{v^{d / 2}} .
$$

We shall now take $d=3$ (because we want to use $L_{03}<\infty$ ) and assume that $L_{13}<C_{3}$. (It has been conjectured that $L_{13}=\frac{1}{15 \pi^{2}}$ by Lieb and Thirring, this satisfies our assumption since $C_{3}=\frac{1}{6 \pi^{2}}$.)

Given the integer $n>0$ we choose $\lambda=\lambda(\mathbf{v}, n)$ such that

$$
\left.\begin{array}{ll}
\lambda=0 & \text { if } n \leqq L_{03} \int_{\Omega} d x \frac{w^{3 / 2}}{v^{3 / 2}} \\
n=L_{03} \int_{\Omega} d x \frac{(w+\lambda)^{3 / 2}}{v^{3 / 2}} & \text { otherwise. }
\end{array}\right\}
$$


We have thus, using (1.7), (2.1), and (2.3),

$$
\begin{aligned}
\sum_{k=1}^{3 n} \mu^{(k)}(\varrho) & \leqq \int \varrho(d \mathbf{v}) \sum_{k=1}^{3 n} a^{(k)}(\mathbf{v}) \leqq \int \varrho(d \mathbf{v}) 3 \sum_{l=1}^{n} b^{(l)}(\mathbf{v}) \leqq 3 \int \varrho(d \mathbf{v}) \sum_{l: b^{(l)}+\lambda \geqq 0} b^{(l)} \\
& \leqq 3 L_{13} \int \varrho(d \mathbf{v}) \int_{\Omega} d x \frac{(w+\lambda)^{5 / 2}}{v^{3 / 2}}-3 C_{3} \int \varrho(d \mathbf{v})|\Omega| \frac{\lambda^{5 / 2}}{v^{3 / 2}}
\end{aligned}
$$

The right hand side is negative provided

$$
\left[\int \varrho(d \mathbf{v}) \int_{\Omega} \frac{d x}{|\Omega|}(w+\lambda)^{5 / 2}\right]^{2 / 5}<\left(\frac{C_{3}}{L_{13}}\right)^{2 / 5}\left(\int \varrho(d \mathbf{v}) \lambda^{5 / 2}\right)^{2 / 5},
$$

or provided

$$
\left(\int \varrho(d \mathbf{v}) \frac{d x}{|\Omega|} w^{5 / 2}\right)^{2 / 5}<\left[\left(\frac{C_{3}}{L_{13}}\right)^{2 / 5}-1\right]\left(\int \varrho(d \mathbf{v}) \lambda^{5 / 2}\right)^{2 / 5} .
$$

In view of (2.4) we have

so that

$$
L_{03}^{-2 / 3} v\left(\frac{n}{|\Omega|}\right)^{2 / 3} \leqq\left(\int \frac{d x}{|\Omega|}(w+\lambda)^{3 / 2}\right)^{2 / 3} \leqq\left(\int \frac{d x}{|\Omega|} w^{3 / 2}\right)^{2 / 3}+\lambda
$$

$$
L_{03}^{-2 / 3} v\left(\frac{n}{|\Omega|}\right)^{2 / 3} \leqq\left(\int \varrho(d \mathbf{v}) \frac{d x}{|\Omega|} w^{5 / 2}\right)^{2 / 5}+\left(\int \varrho(d \mathbf{v}) \lambda^{5 / 2}\right)^{2 / 5}
$$

and therefore (2.5) holds provided

$$
\left(\int \varrho(d \mathbf{v}) \frac{d x}{|\Omega|} w^{5 / 2}\right)^{2 / 5}<\left[\left(\frac{C_{3}}{L_{13}}\right)^{2 / 5}-1\right]\left(L_{03}^{-2 / 3} v\left(\frac{n}{|\Omega|}\right)^{2 / 3}-\left(\int \varrho(d \mathbf{v}) \frac{d x}{|\Omega|} w^{5 / 2}\right)^{2 / 5}\right)
$$

which can be rewritten

$$
L_{03}\left[1-\left(\frac{L_{13}}{C_{3}}\right)^{2 / 5}\right]^{-3 / 2}\left(\int \varrho(d \mathbf{v}) \frac{d x}{|\Omega|} w^{5 / 2}\right)^{3 / 5}<v^{3 / 2} \frac{n}{|\Omega|} .
$$

In conclusion, if $N$ is the smallest integer such that $\sum_{1}^{N} \mu^{(k)}(\varrho)<0$, we have

$$
\limsup _{\Omega \rightarrow \infty} \frac{N}{|\Omega|} \leqq 3 L_{03}\left[1-\left(\frac{L_{13}}{C_{3}}\right)^{2 / 5}\right]^{-3 / 2} v^{-3 / 2}\left(\int \varrho(d \mathbf{v}) \frac{d x}{|\Omega|} w^{5 / 2}\right)^{3 / 5} .
$$

2.1. Theorem. Let @ be an invariant probability measure for the Navier-Stokes time evolution in a bounded region $\Omega \subset \mathbb{R}^{d}, d=2$ or 3 , with regularity conditions stated in Sect. 1. We denote by $|\Omega|$ the $d$-volume of $\Omega$. If $d=3$ we assume that $\varrho$ has compact support in a domain where time evolution is well defined. Let $\mu^{(1)}(\varrho) \geqq \mu^{(2)}(\varrho) \geqq \ldots$ be the characteristic exponents defined in Sect. 1.

(a) For every $\gamma \geqq 1$, the sum of powers of the positive characteristic exponents satisfies

$$
\begin{aligned}
\sum_{k: \mu^{(k)}(\varrho) \geqq 0}\left(\mu^{(k)}(\varrho)\right)^{\gamma} & \leqq \int \varrho(d \mathbf{v}) \sum_{k: \mu^{(k)}(\mathbf{v}) \geqq 0}\left(\mu^{(k)}(\mathbf{v})\right)^{\gamma} \\
& \leqq K_{\gamma d} v^{-\gamma / 2-3 d / 4} \int \varrho(d \mathbf{v}) \int_{\Omega} d x \varepsilon_{\mathbf{v}}(x)^{\gamma / 2+d / 4}
\end{aligned}
$$


where

$$
\varepsilon_{\mathbf{v}}(x)=\frac{v}{2} \sum_{i, j}\left(\frac{\partial v_{i}}{\partial x_{j}}+\frac{\partial v_{j}}{\partial x_{i}}\right)^{2}
$$

is the rate of energy dissipation per unit volume, and the $K_{\gamma d}$ are universal constants.

(b) Let $d=3$ and assume $L_{13}<C_{3}$ (see above). If $N(\varrho)$ denotes the smallest number such that $\sum_{1}^{N(\varrho)} \mu^{(k)}(\varrho)<0$, then

$$
\limsup _{\Omega \rightarrow \infty} \frac{N(\varrho)}{|\Omega|} \leqq K v^{-9 / 4}\left(\int \varrho(d x) \frac{d x}{|\Omega|} \varepsilon_{\mathbf{v}}(x)^{5 / 4}\right)^{3 / 5}
$$

where $K$ is a universal constant ${ }^{7}$.

The first part of (2.7) is just convexity of $t \rightarrow \max \left\{0, t^{\gamma}\right\}$. To proceed we denote by $n(\mathbf{v}, \alpha)$ [respectively $m(\mathbf{v}, \alpha)]$ the number of characteristic exponents $\mu^{(k)}(\mathbf{v})$ [respectively of eigenvalues $\left.a^{(k)}(\mathbf{v})\right]$ which are $\geqq \alpha$. Assuming first $\gamma>1$, we have

$$
\begin{aligned}
\sum_{k: \mu^{(k)}(\mathbf{v}) \geqq 0}\left(\mu^{(k)}(\mathbf{v})\right)^{\gamma} & =\gamma \int_{0}^{\infty} \alpha^{\gamma-1} n(\mathbf{v}, \alpha) d \alpha=\gamma(\gamma-1) \int_{0}^{\infty} \alpha^{\gamma-2} d \alpha \int_{\alpha}^{\infty} n(\mathbf{v}, \beta) d \beta \\
& =\gamma(\gamma-1) \int_{0}^{\infty} \alpha^{\gamma-2} d \alpha \sum_{k=1}^{n(\mathbf{v}, \alpha)}\left(\mu^{(k)}(\mathbf{v})-\alpha\right) .
\end{aligned}
$$

Therefore, using (1.7), we obtain

$$
\begin{aligned}
\int \varrho(d \mathbf{v}) \sum_{k: \mu^{(k)}(\mathbf{v}) \geqq 0}\left(\mu^{(k)}(\mathbf{v})\right)^{\gamma} & \leqq \gamma(\gamma-1) \int_{0}^{\infty} \alpha^{\gamma-2} d \alpha \int \varrho(d \mathbf{v}) \sum_{k=1}^{n(\mathbf{v}, \alpha)}\left(a^{(k)}(\mathbf{v})-\alpha\right) \\
& \leqq \gamma(\gamma-1) \int_{0}^{\infty} \alpha^{\gamma-2} d \alpha \int \varrho(d \mathbf{v}) \sum_{k=1}^{m(\mathbf{v}, \alpha)}\left(a^{(k)}(\mathbf{v})-\alpha\right) \\
& =\int \varrho(d \mathbf{v}) \sum_{k: a^{(k)}(\mathbf{v}) \geqq 0}\left(a^{(k)}(\mathbf{v})\right)^{\gamma} .
\end{aligned}
$$

The resulting inequality is of course also true for $\gamma=1$. Using (2.2) we have thus

$$
\int \varrho(d \mathbf{v}) \sum_{k: \mu^{(k)}(\mathbf{v}) \geqq 0}\left(\mu^{(k)}(\mathbf{v})\right)^{\gamma} \leqq 3 L_{\gamma d} \int \varrho(d \mathbf{v}) \int_{\Omega} d x \frac{w^{\gamma+d / 2}}{v^{d / 2}}
$$

for all $\gamma \geqq 1$, and (2.7) is obtained by using

$$
w^{2} \leqq \frac{1}{4} \sum_{i, j}\left(\frac{\partial v_{i}}{\partial x_{j}}+\frac{\partial v_{j}}{\partial x_{i}}\right)^{2}
$$

Using this same formula we obtain (2.8) from (2.6).

7 We may take $K=3 L_{03}\left[1-\left(\frac{L_{13}}{C_{3}}\right)^{2 / 5}\right]^{-3 / 2} \cdot 2^{-3 / 4}$. The conjectures of Lieb and Thirring give $L_{03}=4 \pi^{-2} 3^{-3 / 2}$ and $L_{13} / C_{3}=2 / 5$, hence $K=0.8185156 \ldots$ 
2.2. Corollary. The Kolmogorov-Sinai (KS) invariant ${ }^{8} h(\varrho)$ satisfies

$$
\begin{aligned}
h(\varrho) & \leqq \int \varrho(d \mathbf{v}) \sum_{k: \mu^{(k)}(\mathbf{v}) \geqq 0} \mu^{(k)}(\mathbf{v}) \\
& \leqq K_{1 d} v^{-1 / 2-3 d / 4} \int \varrho(d \mathbf{v}) d x \varepsilon_{\mathbf{v}}(x)^{1 / 2+d / 4} .
\end{aligned}
$$

The inequality between KS invariant and characteristic exponents is proved in Ruelle [25]; this proof is for finite dimension but the result extends to infinite dimension (Mañé, private communication). If $h(\varrho)>0$, the (turbulent) viscous fluid acts as a source of information (random number generator) ${ }^{9}$, and (2.9) relates the information production and the energy dissipation. In particular, if $d=2$, the rate of information production is bounded above by a multiple of the rate of energy dissipation (the proportionality constant is $K_{12} v^{-2}$ ).

2.3. Corollary. Let $d=3$, and $A$ be a compact attracting set. This means that $A$ is invariant under time evolution and has an open neighborhood $U$ such that if the initial condition is in $U$ the time evolution is defined for all positive times, and asymptotic to $A$. Then, the Hausdorff dimension $H D(A)$ satisfies

$$
\lim _{\Omega \rightarrow \infty} \frac{H D(A)}{|\Omega|} \leqq K v^{-9 / 4} \sup _{\varrho: \operatorname{supp} \varrho \subset A}\left(\int \varrho(d \mathbf{v}) \frac{d x}{|\Omega|} \varepsilon_{\mathbf{v}}(x)^{5 / 4}\right)^{3 / 5} .
$$

This follows from Douady and Oesterlé [4] and Ledrappier [12]. The finiteness of $H D(A)$ follows already from Mallet-Paret [16] or Mañé [18] (see also Foias and Temam [6]).

\section{Classical Approximation Estimate of the Distribution of Characteristic Exponents}

The conjectures of Lieb and Thirring [14] mentioned in Sect. 2 consist, for part of the cases, in replacing a quantum mechanical expression by a classical approximation (see Reed and Simon [24, IV, Sect. XIII.15]). In this section, we directly compute a classical approximation for the density $\frac{d n}{d a}$ of eigenvalues of $\mathscr{H}(v)$. Applying $\int \varrho(d \mathbf{v})$ will give an approximation of the density of characteristic exponents. We derive the relevant formulae, but do not discuss their validity.

Let $P(p)$ be the orthogonal projection on the vector $p \in \mathbb{R}^{d}$, and $Q(p)=$ identity $-P(p)$. We denote by $A(x)$ the matrix with elements $\frac{1}{2}\left(\partial v_{i} / \partial x_{j}+\partial v_{j} / \partial x_{i}\right)$. If $d=2$, $Q A(x) Q$ has two eigenvalues: 0 and $\sigma_{1}(x, p)=-(p, A(x) p) /(p, p)$. If $d=3, Q A(x) Q$ has three eigenvalues: 0 and $\sigma_{1}(x, p) \geqq \sigma_{2}(x, p)$. The classical approximation for the density of eigenvalues of $\mathscr{H}(\mathbf{v})$ is

$$
\frac{d n}{d a}=\frac{1}{(2 \pi)^{d}} \int_{\Omega} d x \int_{\mathbb{R}^{d}} d p \sum_{i=1}^{d-1} \delta\left(v p^{2}+\sigma_{i}(x, p)+a\right) .
$$

\footnotetext{
8 The Kolmogorov-Sinai invariant is also called entropy; this entropy corresponds to time evolution and is not related to the thermodynamic entropy of the fluid

9 For a nice discussion of this point see Shaw [30]
} 
In the case $d=2$, we write

$$
\sigma(x)=\left(\frac{1}{4} \sum_{i, j}\left(\frac{\partial v_{i}}{\partial x_{j}}+\frac{\partial v_{j}}{\partial x_{i}}\right)^{2}\right)^{1 / 2}
$$

so that the eigenvalues of $A(x)$ are $\pm \sigma(x)$, and

$$
\begin{aligned}
\int_{\mathbb{R}^{2}} d p \delta\left(v p^{2}+\sigma_{1}(x, p)+a\right) & =\int r d r d \theta \delta\left(v r^{2}-\sigma(x) \cos 2 \theta+a\right) \\
& =\frac{1}{2 v} \operatorname{meas}\{\theta \in[0,2 \pi]: \sigma(x) \cos 2 \theta-a>0\} \\
& =\frac{1}{v} \operatorname{meas}\left\{\theta \in\left[-\frac{\pi}{2}, \frac{\pi}{2}\right]: \sin \theta>\frac{a}{\sigma(x)}\right\} \\
& =\frac{1}{v} \operatorname{meas}\left\{\theta \in\left[-\frac{\pi}{2}, \frac{\pi}{2}\right]: \theta>\phi\left(\frac{a}{\sigma(x)}\right)\right\},
\end{aligned}
$$

where

$$
\phi(\xi)=\left\{\begin{array}{lll}
-\frac{\pi}{2} & \text { if } \quad \xi<-1 \\
\arg \sin \xi & \text { if } \quad|\xi| \leqq 1 \\
\frac{\pi}{2} & \text { if } \quad \xi>1
\end{array}\right.
$$

Therefore

$$
\int_{\mathbb{R}^{2}} d p \delta\left(v p^{2}+\sigma_{1}(x, p)+a\right)=\frac{1}{v}\left(\frac{\pi}{2}-\phi\left(\frac{a}{\sigma(x)}\right)\right) .
$$

Finally

$$
|\Omega|^{-1} \frac{d n}{d a}=\frac{1}{4 \pi} \cdot \frac{1}{v}\left(\frac{1}{2}-\frac{\langle\phi(a / \sigma)\rangle}{\pi}\right),
$$

where $\langle\ldots\rangle$ denotes an average over $\sigma=\sigma(x)$ corresponding to $|\Omega|^{-1} \int d x$. If we approximate the characteristic exponents $\mu^{(k)}$ by the eigenvalues of $\mathscr{H},(3.1)$ may be interpreted as an estimate for the density of characteristic exponents. An average over $\mathbf{v}$ corresponding to $\int \varrho(d \mathbf{v})$ has then to be understood. Notice that (3.1) interpolates between $(4 \pi v)^{-1}$ for $a \rightarrow-\infty$ and 0 for $a \rightarrow \infty$; the interpolation is symmetric around $a=0$.

In the case $d=3$ we have

$$
\int_{\mathbb{R}^{3}} d p \sum_{i=1}^{2} \delta\left(v p^{2}+\sigma_{i}(x, p)+a\right)=v^{-3 / 2} \psi(A+a) .
$$

We have written

$$
\psi(A+a)=\frac{1}{2} \sum_{i=1}^{2} \int \sin \theta d \theta d \varphi\left(-\sigma_{i}(x, u(\theta, \varphi))-a\right)^{1 / 2} \Theta\left(-\sigma_{i}(x, u(\theta, \varphi))-a\right)
$$

where $u(\theta, \varphi)=(\sin \theta \cos \varphi, \sin \theta \sin \varphi, \cos \theta)$ and $\Theta(t)=\frac{1}{2}(1+\operatorname{sgn} t)$. The eigenvalues $\sigma_{1}(x, u), \sigma_{2}(x, u)$ are the roots of the equation

$$
\sigma^{2}+(u, A u) \sigma+\left(u, A^{2} u\right)-\frac{1}{2} \operatorname{Tr} A^{2}=0 .
$$


By rotational invariance, $\psi(A+a)$ depends only on the spectrum of $A+a$. Furthermore, $\psi(A+a)$ is homogeneous of degree $\frac{1}{2}$ in its argument, and decreasing. Clearly

$$
\psi \approx\left\{\begin{array}{lll}
4 \pi \sqrt{|a|} & \text { for } & a \rightarrow-\infty \\
0 & \text { for } & a \rightarrow \infty
\end{array}\right.
$$

We obtain thus

$$
\begin{aligned}
|\Omega|^{-1} \frac{d n}{d a} & =\frac{1}{(2 \pi)^{3}} \cdot \frac{1}{v^{3 / 2}}\langle\psi(A+a)\rangle \\
& \approx \begin{cases}v^{-3 / 2} \cdot\left(2 \pi^{2}\right)^{-1}|a|^{1 / 2} & \text { for } a \rightarrow-\infty, \\
0 & \text { for } a \rightarrow \infty .\end{cases}
\end{aligned}
$$

Again we have used $\langle\ldots\rangle$ in (3.2) to denote the average $|\Omega|^{-1} \int d x$ [and $\left.\int \varrho(d \mathbf{v})\right]$. To obtain more information on $\frac{d n}{d a}$, it is thus necessary to study the statistics of $A$. Some numerical information on the moments is known for the case of isotropic turbulence ${ }^{10}$.

\section{Distribution of Characteristic Exponents in an Intermittent Model of Turbulence $(d=3)$}

The experimental study of developed turbulence shows that the velocity gradient takes large values on only a small fraction of the domain $\Omega$. We may write

$$
\frac{\partial v_{i}}{\partial x_{j}}=\frac{1}{2}\left(\frac{\partial v_{i}}{\partial x_{j}}-\frac{\partial v_{j}}{\partial x_{i}}\right)+\frac{1}{2}\left(\frac{\partial v_{i}}{\partial x_{j}}+\frac{\partial v_{j}}{\partial x_{i}}\right)
$$

Therefore the vorticity $(=\operatorname{curl} v)$ and the dissipation $\left(\varepsilon=\frac{v}{2} \sum_{i, j}\left(\frac{\partial v_{i}}{\partial x_{j}}+\frac{\partial v_{j}}{\partial x_{i}}\right)^{2}\right)$ are concentrated on a small fraction of physical $d$-dimensional volume. This is the phenomenon of intermittency. It may be modelled by assuming that the fraction of volume occupied by "eddies of size $l, " 11$ per unit interval of $\log l$, is given by a function $\beta$ of $l$. We suppose that $\Omega$ is large and that energy is fed into the fluid at a wavelength $L$ (large eddy size). Usually $L$ is of the order of the diameter of $\Omega$ and the volume of $\Omega$ is $|\Omega| \sim L^{3}$. A small eddy size $\eta$ will correspond to dissipation. The following is then a natural choice for $\beta$ :

$$
\beta(l)= \begin{cases}(l / L)^{\alpha} & \text { for } L \geqq l \geqq \eta \text { (inertial range) } \\ 0 & \text { otherwise }\end{cases}
$$

where $\alpha>0$ denotes a (universal) constant.

Frisch et al. [7] following Novikov and Steward [21], Mandelbrot [17], and ideas of Kraichnan [10] have obtained a dynamical model of turbulence along the above lines. This " $\beta$-model" generalizes the Kolmogorov theory of turbulence to

10 See Betchov [1] and Siggia [31]. I am indebted to Eric Siggia for indicating these references to me 11 The concept of eddy has varied between that of a rather well defined localized object, to that of a mere label in Fourier analysis. The former view is accepted here 
allow for intermittency. We now briefly paraphrase the ideas of Frisch, Sulem, and Nelkin. Throughout what follows we take $d=3$.

As in the Kolmogorov theory, the energy cascades from large to small lengths. Let $\varepsilon$ be the mean energy dissipation (or injection, or transfer) per unit time and unit mass. One introduces a characteristic time $\tau(l)$ (turnover time for eddies of size $l$ ) and a characteristic velocity $v(l)$ (velocity variation over distance $l$ in active region). These are related by the following natural conditions

$$
\begin{gathered}
v(l) \sim l / \tau(l), \\
\varepsilon \sim l(\beta) \cdot v(l)^{2} / \tau(l) \text { for } l \leqq \eta
\end{gathered}
$$

(where $\sim$ denotes approximate proportionality). The fact that the same $\tau(l)$ occurs in (4.2) and (4.3) is a crucial dynamical assumption. The dissipation is naturally given by

so that $\eta$ satisfies

$$
\varepsilon \sim \beta(\eta) \times \frac{v(\eta)^{2}}{\eta^{2}}
$$

$$
\tau(\eta) \sim \eta^{2} / v
$$

From (4.1), (4.2), (4.3), and (4.4) one obtains

$$
\left.\begin{array}{c}
\tau(l) \sim l^{\frac{2+\alpha}{3}} L^{-\alpha / 3} \varepsilon^{-1 / 3} \\
\eta \sim\left(\frac{\nu^{3}}{\varepsilon}\right)^{\frac{1}{4-\alpha}} L^{-\frac{\alpha}{4-\alpha}} \\
\tau(\eta) \sim \varepsilon^{-\frac{2}{4-\alpha}} \varepsilon^{\frac{2+\alpha}{4-\alpha}} L^{-\frac{2 \alpha}{4-\alpha}} .
\end{array}\right\}
$$

We shall here identify the number $d n$ of characteristic exponents in the range $(a, a+d a)$ with the number of eigenvalues $a^{(k)}(\mathbf{v})$ of $\mathscr{H}(\mathbf{v})$ in this range (see Sect. 1). The classical approximation (see Reed and Simon [24, IV, Sect. XIII.15]) gives:

$d n \sim$ phase space volume of the set of points $(x, p)$ such that

$$
-v p^{2}+V(x) \in(a, a+d a)
$$

where we have

Thus

$$
V(x) \sim\left(\sum_{i, j}\left(\frac{\partial v_{i}}{\partial x_{j}}+\frac{\partial v_{j}}{\partial x_{i}}\right)^{2}\right)^{1 / 2}
$$

$$
\frac{d n}{d a} \sim \int_{x: V(x)>a} d^{3} x d^{3} p \delta\left(-v p^{2}+V(x)-a\right)=\int_{x: V(x)>a} d^{3} x \frac{4 \pi p^{2}(a, x)}{2 v p(a, x)}
$$

where $p(a, x)=\left(\frac{V(x)-a}{v}\right)^{1 / 2}$. Hence

$$
\frac{d n}{d a} \sim|\Omega| \int_{\eta}^{l(a)} \beta(l) \frac{d l}{l} \cdot v^{-3 / 2}\left(\tau(l)^{-1}-a\right)^{1 / 2},
$$

where $l(a)$ is determined by $\tau(l(a))=a^{-1}$. 
In view of (4.1) and (4.5) we have

$$
\beta \sim \varepsilon^{\frac{\alpha}{2+\alpha}} L^{-\frac{2 \alpha}{2+\alpha}} \frac{3 \alpha}{2+\alpha}
$$

for $\tau \leqq \tau(L)$ so that, for $a \geqq \tau(L)^{-1}$,

$$
\begin{aligned}
|\Omega|^{-1} \frac{d n}{d a} & \sim v^{-3 / 2} a^{1 / 2} \int\left((a \tau)^{-1}-1\right)^{1 / 2} d \beta \\
& \sim v^{-3 / 2} a^{1 / 2}\left(\frac{\varepsilon}{a^{3} L^{2}}\right)^{\frac{\alpha}{2+\alpha}} \int\left((a \tau)^{-1}-1\right)^{1 / 2} d(a \tau)^{\frac{3 \alpha}{2+\alpha}} \\
& =v^{-3 / 2} a^{1 / 2}\left(\frac{\varepsilon}{a^{3} L^{2}}\right)^{\frac{\alpha}{2+\alpha}} I
\end{aligned}
$$

where

$$
I=\int_{a \tau(\eta)}^{1} \sqrt{1-\xi} \cdot \xi^{-\frac{3(2-\alpha)}{2(2+\alpha)}} d \xi
$$

We may thus write

$$
\begin{aligned}
|\Omega|^{-1} d n & \sim I \cdot\left(\frac{\varepsilon}{v^{3} L^{2}}\right)^{1 / 2} d\left(\frac{a^{3} L^{2}}{\varepsilon}\right)^{\frac{2-\alpha}{2(2+\alpha)}} \\
& \sim I \cdot L^{-1-\frac{\alpha}{2}} \eta^{-2+\frac{\alpha}{2}} d(a \tau(L))^{\frac{3(2-\alpha)}{2(2+\alpha)}} \\
& \sim I \cdot \beta(\eta) \eta^{-3} d(a \tau(\eta))^{\frac{3(2-\alpha)}{2(2+\alpha)}}
\end{aligned}
$$

Clearly, $I=0$ for $a \geqq \tau(\eta)^{-1}$. To estimate $I$ for $a \ll \tau(\eta)^{-1}$ we distinguish 3 cases :

(i) if $\alpha>2 / 5$, i.e. $\frac{3(2-\alpha)}{2(2+\alpha)}<1, I \approx$ const,

(ii) if $\alpha=2 / 5$, i.e. $\frac{3(2-\alpha)}{2(2+\alpha)}=1, I \approx \log (a \tau(\eta))^{-1}$,

(iii) if $\alpha<2 / 5$, i.e. $\frac{3(2-\alpha)}{2(2+\alpha)}>1, I \sim(a \tau(\eta))^{-\frac{2-5 \alpha}{2(2+\alpha)}}$.

Therefore

$$
\begin{aligned}
|\Omega|^{-1} \frac{d \eta}{d a} & \sim\left\{\begin{array}{ll}
\beta(\eta) \eta^{-3} \tau(\eta) J(a) & \text { for } \quad a \tau(\eta)<1 \\
0 & \text { for } \quad a \tau(\eta) \geqq 1
\end{array},\right. \\
J(a) & \sim \begin{cases}(a \tau(\eta))^{\frac{2-5 \alpha}{2(2+\alpha)}} & \text { for } \quad \alpha>2 / 5 \\
\log (a \tau(\eta))^{-1} & \text { for } \quad \alpha=2 / 5 . \\
\text { constant } & \text { for } \quad \alpha<2 / 5\end{cases}
\end{aligned}
$$

In all these cases, the moments of the distribution of positive characteristic exponents satisfy $|\Omega|^{-1} \int a^{k} d n \sim \beta(\eta) \eta^{-3} \tau(\eta)^{-k}$. In particular, the number of char- 
acteristic exponents per unit volume is $\frac{n}{|\Omega|} \sim \beta(\eta) \eta^{-3}$. This is the natural expression, reducing to $\eta^{-3}$ for the Kolmogorov theory, i.e., for $\alpha=0$ (see Ruelle [27]).

Experimentally, it seems that $\alpha \approx 0.3$ to $0.5^{12}$ so that the value $2 / 5$ is not excluded, and any of the three cases discussed above could conceivably occur.

\section{Some More Remarks}

(a) In an isothermal fluid the dissipation $\varepsilon_{\mathbf{v}}(x)$ is proportional to the rate of entropy production. Our inequalities in Sect. 2 thus imply bounds on the information creation in terms of entropy production. Since entropy production corresponds to loss of microscopic information, this result has some philosophical appeal. Notice however that a real information balance should involve Boltzmann's constant.

(b) The "barber pole" turbulence which fascinated Feynman (Feynman et al. [5, II, Sect. 41-6]) appears in the flow between two concentric rotating cylinders and consists of an helical turbulent band alternating with a "laminar" region. A given element of fluid in the laminar region may enter the turbulent band and leave it again after some time. To interpret this situation one would like to define spatially localized characteristic exponents. Such a notion would also be useful in meteorology (see Leith [13]). Because of the global nature of characteristic exponents, a formal definition of "local characteristic exponents" is not easy to formalize. One can however see what happens physically: a disturbance $\delta v$ of the flow can grow only in a region where the "potential" $w$ of Sect. 2 (this is essentially the dissipation) is sufficiently large. The positive contributions to characteristic exponents come therefore from regions with large dissipation: in other regions turbulence may be damped to a "laminar motion."

(c) We expect the decay of an eddy of size $l$ into smaller eddies to depend sensitively on initial conditions. The characteristic time for decay should thus be the same as the characteristic time for growth of a small perturbation. From this follows that it is reasonable to use the same "turnover-time" $\tau(l)$ in Eqs. (4.2) and (4.3) of the $\beta$-model.

Note that an eigenstate $\psi^{(k)}$ of $\mathscr{H}(\mathbf{v})$ will contribute most to the decay of a certain eddy of size $l$ if the contribution of this eddy to the eigenvalue $a^{(k)}(\mathbf{v})$ is near the maximum [which is $\sim \tau(l)^{-1}$ ]. In the quantum mechanical picture such a state is near the ground state, has simple geometry, and will yield a decay into a small number of eddies of size not much smaller than $l$. In the opposite direction, very excited eigenstates, which correspond to decay of an eddy into a large number of small eddies, are associated with small eigenvalues of $\mathscr{H}(\mathbf{v})$ and therefore long lifetimes, and small contributions to decay.

(d) If we take $\alpha \geqq 2 / 5$ in the $\beta$-model, we find that the density of characteristic exponents per unit volume diverges at $a=0$. This density is therefore infinite for

12 See Monin and Yaglom [20, Vol. 2, Sect. 25] (our parameter $\alpha$ is usually called $\mu$ in the literature). The value $\alpha \approx 0.5 \pm 0.2$ is given by a recent numerical simulation of Brachet [2] (I am indebted to Uriel Frisch for this reference). See also Chorin [3]: $\alpha \approx 0.5$ 
$a \leqq 0$ (because the function $\psi$ of Sect. 3 is decreasing), and there is an infinite number of characteristic exponents $\approx 0$ per unit volume. If we consider this situation unacceptable, we are forced to take $\alpha<2 / 5$.

(e) In the discussions of this paper we have not made a specific choice of the invariant measure $\varrho$. It is in principle possible to find $\varrho$ on the basis of its assumed stability under small stochastic perturbations (see Ruelle [26] and references quoted there). In particular, this should permit the determination of the parameter $\alpha$ in the $\beta$ model (Sect. 4), but the idea seems difficult to implement ${ }^{13}$.

Acknowledgements. Helpful comments by Uriel Frisch and Eric Siggia are gratefully acknowledged.

\section{References}

1. Betchov, R.: An inequality concerning the production of vorticity in isotropic turbulence. J. Fluid Mech. 1, 497-504 (1956)

2. Brachet, M.: Intégration numérique des équations de Navier-Stokes en régime de turbulence développée. C.R. Acad. Sci. Paris 294, Série II, 537-540 (1982)

3. Chorin, A.J.: The evolution of a turbulent vortex. Commun. Math. Phys. 83, 517-535 (1982)

4. Douady, A., Oesterlé, J.: Dimension de Hausdorff des attracteurs. C.R. Acad. Sci. Paris 290 A, 1135-1138 (1980)

5. Feynman, R.P., Leighton, R.B., Sands, M.: The Feynman lectures on physics. Reading, Mass.: Addison-Wesley 1963(I), 1964(II), 1965(III)

6. Foias, C., Temam, R.: Some analytic and geometric properties of the solutions of the evolution Navier-Stokes equations. J. Math. Pure Appl. 58, 339-368 (1979)

7. Frisch, U., Sulem, P.-L., Nelkin, M.: A simple dynamical model of intermittent fully developed turbulence. J. Fluid Mech. 87, 719-736 (1978)

8. Fujisaka, H., Mori, H.: A maximum principle for determining the intermittency exponent of fully developed steady turbulence. Progr. Theor. Phys. 62, 54-60 (1979)

9. Girault, V., Raviart, P.-A.: Finite element approximation of the Navier-Stokes equations. In: Lecture Notes in Mathematics, Vol. 749. Berlin, Heidelberg, New York: Springer 1979

10. Kraichnan, R.H.: On Kolmogorov's inertial-range theories. J. Fluid Mech. 62, 305-330 (1974)

11. Ladyzhenskaya, O.A.: The mathematical theory of viscous incompressible flow (2nd ed.). Moscow: Nauka, 1970 [2nd english ed. New York: Gordon and Breach 1969]

12. Ledrappier, F.: Some relations between dimension and Lyapounov exponents. Commun. Math. Phys. 81, 229-238 (1981)

13. Leith, C.: Chaos and order in wheather prediction. Order in chaos (Los Alamos, 1982) NorthHolland (to be published)

14. Lieb, E., Thirring, W.: Inequalities for the moments of the eigenvalues of the Schrödinger equation and their relation to Sobolev inequalities. In : Studies in Mathematical Physics: Essays in honor of Valentine Bargman, Lieb, E., Simon, B., Wightman, A.S. (eds.), pp. 269-303. Princeton, NJ: Princeton University Press 1976

15. Lions, J.L.: Quelques méthodes de résolution des problèmes aux limites non-linéaires. Paris 1969

16. Mallet-Paret, J.: Negatively invariant sets of compact maps and an extension of a theorem of Cartwright. J. Diff. Eq. 22, 331-348 (1976)

17. Mandelbrot, B.: Intermittent turbulence and fractal dimension : kurtosis and the spectral exponent $5 / 3+B$. In: Turbulence and the Navier-Stokes equations. Lecture Notes in Mathematics, Vol. 565, pp. 121-145. Berlin, Heidelberg, New York: Springer 1976

18. Mañé, R.: On the dimension of the compact invariant sets of certain nonlinear maps (preprint)

19. Mañé, R.: Lyapounov exponents and stable manifolds for compact transformations (preprint)

20. Monin, A.S., Yaglom, A.M.: Statistical fluid mechanics: mechanics of turbulence. Moscow: Nauka, 1965 [english transl.: 2 Vol. (ed. by J. L. Lumley). Cambridge, Mass. : MIT Press 1971 and 1975]

13 An ad hoc information-theoretical variational principle has been used by Fujisaka and Mori [8] and leads to $\alpha \approx 0.341$ 
21. Novikov, E.A., Steward, R.W.: Intermittency of turbulence and the spectrum of fluctuations of energy dissipation. Izv. Akad. Nauk SSSR Ser. Geofiz. 3, 408-413 (1964)

22. Oseledec, V.I. : A multiplicative ergodic theorem. Lyapunov characteristic numbers for dynamical systems. Tr. Moskov Mat. Obšč. 19, 179-210 (1968) [english transl.: Transl. Moscow Math. Soc. 19, 197-221 (1968)]

23. Raghunathan, M.S.: A proof of Oseledec' multiplicative ergodic theorem. Israel J. Math. 32, 356-362 (1979)

24. Reed, M., Simon, B.: Methods of modern mathematical physics. New York: Academic Press 1972(I), 1975(II), 1979(III), 1978(IV)

25. Ruelle, D.: An inequality for the entropy of differentiable maps. Bol. Soc. Bras. Mat. 9, 83-87 (1978)

26. Ruelle, D.: What are the measures describing turbulence? Progr. Theor. Phys. Suppl. 64, 339-345 (1978)

27. Ruelle, D.: Microscopic fluctuations and turbulence. Phys. Lett. 72 A, 81-82 (1979)

28. Ruelle, D.: Measures describing a turbulent flow. Ann. N.Y. Acad. Sci. 357, 1-9 (1980)

29. Ruelle, D.: Characteristic exponents and invariant manifolds in Hilbert space. Ann. Math. 115, 243-290 (1982)

30. Shaw, R.: Strange attractors, chaotic behavior, and information flow. Santa Cruz Preprint (1977)

31. Siggia, E.: Invariants for the one-point vorticity and strain rate correlation functions. Phys. Fluids 24, 1934-1936 (1981)

32. Sinai, Ya.G.: On the entropy per particle for the dynamical system of hard spheres (preprint)

33. Temam, R.: Navier-Stokes equations. Revised edition. Amsterdam: North-Holland 1979

Communicated by A. Jaffe

Received August 24, 1982 